\title{
Theoretical and Experimental Study on Ultrasonic-Mechanical Plating
}

\author{
Zongwei Niu*, Li Li, Guangming Zheng, Xiuting Wei and Zhiyong Li \\ School of Mechanical Engineering, Shandong University of Technology, Zibo 255049, China \\ ${ }^{*}$ Corresponding author
}

\begin{abstract}
The traditional mechanical plating method is not suitable for parts of big bulk and of complex surface. In order to overcome above shortcoming of mechanical plating, a novel wet ultrasonic mechanical plating process was present by integrating the theories of mechanical plating and ultrasonic shot peening. Wet ultrasonic mechanical plating equipment which includes the ultrasonic generator, ultrasonic transducer and amplitude transformer horn was designed. Galvanized tests were carried out using the equipment. The properties of the plating layer were tested. The results show that plating layer whose maximum thickness can reach $30 \mu \mathrm{m}$ can be gotten on the surface of workpiece. The bonding strength between the coating and substrate is satisfactory.
\end{abstract}

Keywords - mechanical plating; ultrasonic vibration; ultrasonic transducer; amplitude transformer horn

\section{INTRODUCTION}

Mechanical plating is a kind of surface treatment process in which the workpiece as well as metal powders, impingement medium (normally are glass beads), dispersant, accelerant and liquid medium are put into a rotary cylinder, a flow circumstance of impact, rub and grind is formed as the cylinder rotate, therefore the metal powders form plating layer on the surface of workpiece by the effect of mechanical impact and physical and chemical deposition at room temperature and atmospheric pressure [1].

Compared with hot-dip coating and electroplating, mechanical plating has following merits:

Firstly, there is no hydrogen embrittlement and annealing softening after mechanical plating. The hydrogen is evolved during electroplating of steel workpiece which results in hydrogen embrittlement and stress concentration that deteriorates the product performance seriously. There is no hydrogen evolved during mechanical plating which can avoid the hydrogen embrittlement. The high temperature of hot-dip coating may cause the annealing softening of workpiece which also influence the product performance. Mechanical plating is carried out at room temperature which can avoid the annealing softening.

Secondly, the process of mechanical plating can realize cleaner production. The raw material used in mechanical is nontoxic. The process water can be recycled. Compared with hot-dip coating, in mechanical plating there is no smoke, harmful gas or metal vapor emerged caused by high temperature and hydrogen chloride and ammonia derived from the flux volatilization. And there is no harmful gas generated at the cathode in electroplating. Therefore mechanical plating is prone to green manufacturing or cleaner production [2].

Finally, mechanical plating is of low cost and high productivity. The equipments of mechanical plating are simple and easy to operate. It is suitable for quantity production of small workpiece. The quantity of workpiece being processed by mechanical plating each time can be extremely large and the first-pass yield of products is high.

Unfortunately, the traditional mechanical plating is not suitable for workpiece of big bulk and of complex shape due to the rolling and collision of the workpieces in the rotary drum during the plating process. It's necessary to reform the mechanical plating method to overcome its above shortcoming. Sergey V. Komarov et al presented a novel method for dry mechanical plating using ultrasonic vibrations [3-4]. In this paper we present a novel wet ultrasonic mechanical plating process by integrating the principles of mechanical plating and ultrasonic shot peening.

\section{STUDY OF THE THEORY}

During the mechanical plating, tin salt and ferric salt is used to generate driving metals tin and iron or their cations, which can induce zinc to deposit on the substrate surface of the workpiece. The driving metals remain in the plating layer, distribute in the clearance or boundary of the zinc powder particles.

The plating layer is a multiphase mixing system which is composed of plating metal powders, driving metals and clearances. The plating metal powder is the main component. Under the dual effects of mechanical and chemical bonds combination of occlusion, plating layer and substrate are bonded together.

In traditional mechanical shot peening, the high speed balls will generate impact loading as they reaching the workpiece surface. This impact loading can replaced by ultrasonic vibration of the shot pin in ultrasonic shot peening.

The principle of wet ultrasonic mechanical plating is similar to that of ultrasonic shot peening. The ultrasonic vibration system provides power for the impingement medium (glass beads) to produce high speed and peen the metal powders onto the surface of workpiece to form cladding film. 


\section{The Design of Wet Ultrasonic Plating EQUiPMENT}

The schematic diagram of wet ultrasonic plating equipment is shown in Figure I. It mainly includes he ultrasonic generator, ultrasonic transducer, amplitude transformer horn, and plating tank.

The workpiece, metal powders, impingement medium (glass beads), dispersant, accelerant and liquid medium are placed into a resonant plating tank. The tank is set into a high-frequency vibration by using an ultrasonic transducer attached to the tank bottom. This initiates a chaotic motion and collision of the glass beads and the powder particles inside the tank that results in the grinding of the particles, thereby hammering them further into the workpiece surface.

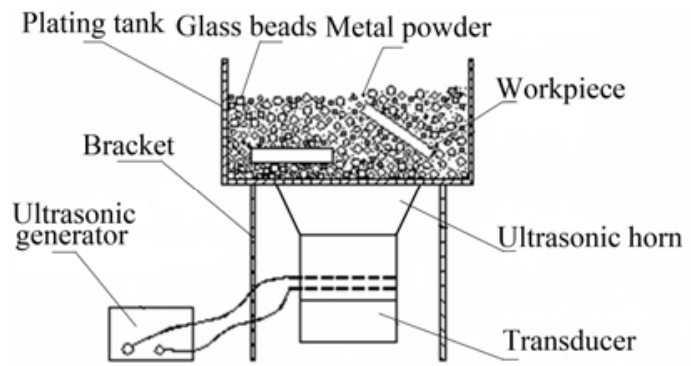

FIGURE I. THE SCHEMATIC DIAGRAM OF WET ULTRASONIC MECHANICAL PLATING EQUIPMENT

\section{A. The Design of Ultrasonic Transducer}

The structure of ultrasonic transducer is shown in Figure II. Piezoelectric ceramic transducer was chosen. In order to satisfy the resonance condition, it is designed that the sum of the thicknesses of rear cover plate, the piezoelectric ceramic slices and electrode equals quarter wavelength of ultrasonic vibration, and the thickness of front cover plate equals quarter wavelength of ultrasonic vibration, i.e. $\mathrm{t}_{1}=63.4 \mathrm{~mm}[5]$.

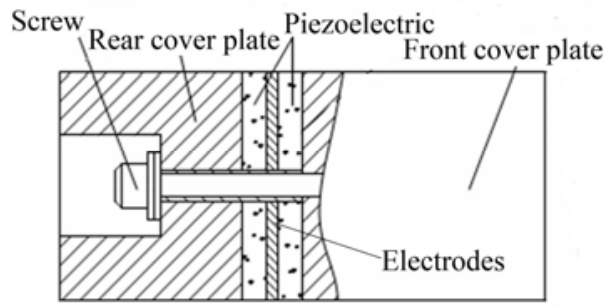

FIGURE II. THE STRUCTURE OF ULTRASONIC TRANSDUCER
The resonance frequency of the ultrasonic transducer is 20 $\mathrm{kHz}$. The front cover plate is of LY12 aluminum material, its diameter is $60 \mathrm{~mm}$, the density $\rho_{1}=2.7 \mathrm{~g} / \mathrm{cm}^{3}$, the propagation speed of ultrasonic vibration in it $c_{1}=5.07 \times 10^{5 \mathrm{~cm}} / \mathrm{s}$. The rear cover plate is of 45 carbon steel material, its diameter is $60 \mathrm{~mm}$, the density $\rho_{3}=7.85 \mathrm{~g} / \mathrm{cm}^{3}$, the propagation speed of ultrasonic vibration in it $c_{3}=5.1 \times 10^{5 \mathrm{~cm} / \mathrm{s}}$. The diameter of piezoelectric ceramic slice is $56 \mathrm{~mm}$, the diameter of its inner hole is $16 \mathrm{~mm}$, the thickness is $6 \mathrm{~mm}$, the density $\rho_{2}=7.5 \mathrm{~g} / \mathrm{cm} 3$, the propagation speed of ultrasonic vibration in it $c_{2}=3.57 \times 10^{5 \mathrm{~cm} / \mathrm{s}}$. The thickness of electrode $t=3 \mathrm{~mm}$, its diameter is $56 \mathrm{~mm}$.

The thickness of rear cover plate is calculated using following equation:

$$
\tan \left(\frac{\omega \mathrm{t}_{3}}{\mathrm{c}_{3}}\right) \tan \left(\frac{\omega \mathrm{t}_{2}}{\mathrm{c}_{2}}\right)=\frac{Z_{2}}{Z_{3}}
$$

Whereas $Z_{2}$ and $Z_{3}$ are the impedances of piezoelectric ceramic slice and rear cover plate respectively.

$$
\frac{Z_{2}}{Z_{3}}=\frac{S_{2} \rho_{2} \mathrm{c}_{2}}{S_{3} \rho_{3} \mathrm{c}_{3}}
$$

Plug given data into above equations, can get:

$$
t_{2}=16 \mathrm{~mm}, t_{3}=15 \mathrm{~mm} \text {. }
$$

\section{B. The Design of Ultrasonic Amplitude Horn}

The vibration amplitude of piezoelectric transducer is very tiny which need to be amplified using horn. The normal shapes of horn include cone, exponential, suspension alignment and step. In order to make the horn has adequate strength and easy to be machined, we choice the horn of cone shape and of half wavelength. The structure of the horn is shown as Figure III. Whereas $l_{1}, l_{2}, l_{3}$ are the lengths of the thick end, the cone section, and the thin end respectively, $D_{1}, D_{2}$ are the diameters of thick end and thin end respectively. Usually $l_{1}$ was selected as quarter wavelength of the ultrasonic in the horn media that makes the nodal surface locates at the boundary of cone and cylinder where the vibration amplitude is the minimum.

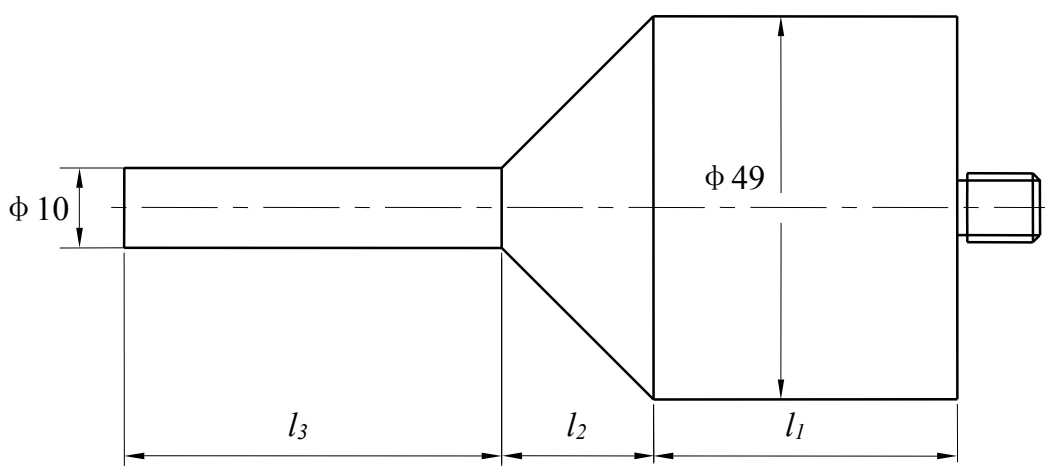

FIGURE III. THE STRUCTURE OF THE HORN 
The frequency equation of cone smooth elastic body is as following:

$$
\tan k l_{3}=\cot k l_{2}+\frac{1}{k l_{2}} \cdot \frac{R_{1}-R_{2}}{R_{2}}
$$

The amplification factor is

$$
M=\frac{R_{1}}{R_{2}} \cdot \frac{\sin k l_{2}}{\cos k l_{3}}
$$

As can be known from above formula, the amplification is determined by $R_{1}, R_{2}, l_{2}$ and $l_{3}$. Moreover, $l_{3}$ is determined by $l_{2}$. Therefore, the dimensions of the hone can be identified provided $R_{1}, R_{2}$ and $l_{2}$ are known. $R_{1}$ is in accordance with the transducer dimension and is a fixed value corresponding to a particular transducer. $R_{2}$ is the end radius of the horn which is variable with the desired vibration amplitude. The length of the cone section $l_{2}$ is inadvisable to be excessively large; otherwise the amplification factor would be too small, normally let $k l_{2}=\frac{\pi}{4}$. In this design, $f=20 \mathrm{kHz}, R_{1}=24.5 \mathrm{~mm}, R_{2}=5 \mathrm{~mm}$.

\section{EXPERIMENT METHODS}

The experiments were carried out using Q235 gasket of the $\varphi 22 \mathrm{~mm} \times \varphi 10 \mathrm{~mm} \times 2 \mathrm{~mm}$ size as substrate workpieces, using zinc powder of 800-grit as coating materials, and using glass bead of 40 80 grit as impingement medium. The chemical materials added into the plating tank include activator (sulphuric acid, citric acid), corrosion inhibitor (ammonium dibasic phosphate, ammonium citrate), deposit agent (stannous sulfate), dispersant (polyethylene glycol) and surfactant (lauryl sodium sulfate) etc. The specimens were taken out and washed to be tested after the ultrasonic mechanical plating had lasted for 2 minutes.

The coating thickness was measured using thickness gauge. Average value of five random points on a specimen was calculated and taken as the result. The adhesive strength was examined using following method. Square grids of $1 \mathrm{~mm}$ on each edge were scratched on the surface of the specimen using $\mathrm{s}$ hard steel slide knife with a cutter edge of 30 degree. When the line is scratched, sufficient pressure should be applied to break the covering layer at one time to reach the base metal. Evaluate the adhesive strength according to whether the coating in the lattice is stripped from the substrate. The surface topography of the coating was observed using stereomicroscope.

\section{EXPERIMENT RESULT AND ANALYSIS}

The thicknesses of the zinc coating on the workpiece surface are shown in Table I. The SEM photographs of the zinc coating surface and the cross section were shown in Figure IV(a) and IV(b) respectively. From above results it can be known that even zinc coating on the workpiece surface can be achieved via wet ultrasonic mechanical plating. The coating layer is smooth and without flaws such as omission plating, peeling and inclusions.

TABLE I. THE THICKNESS OF ZINC-COATING

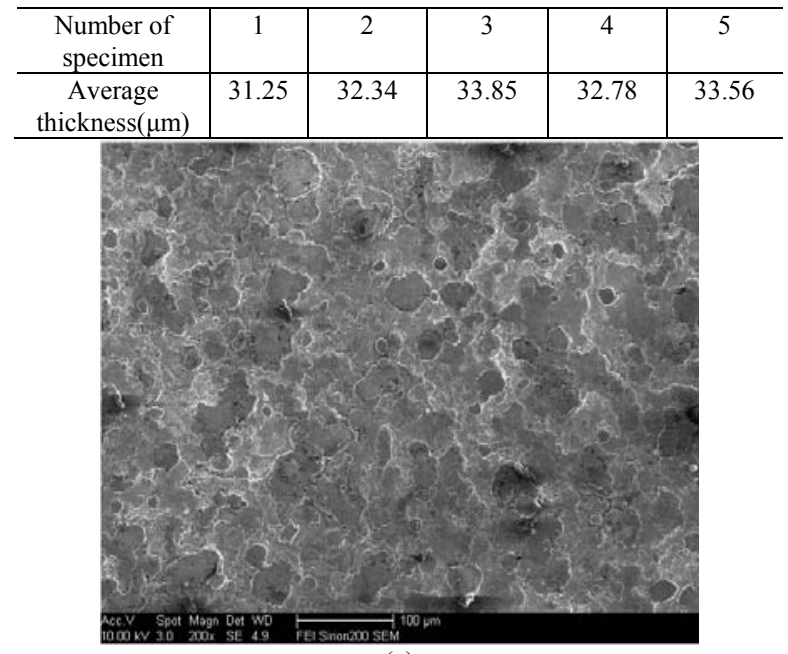

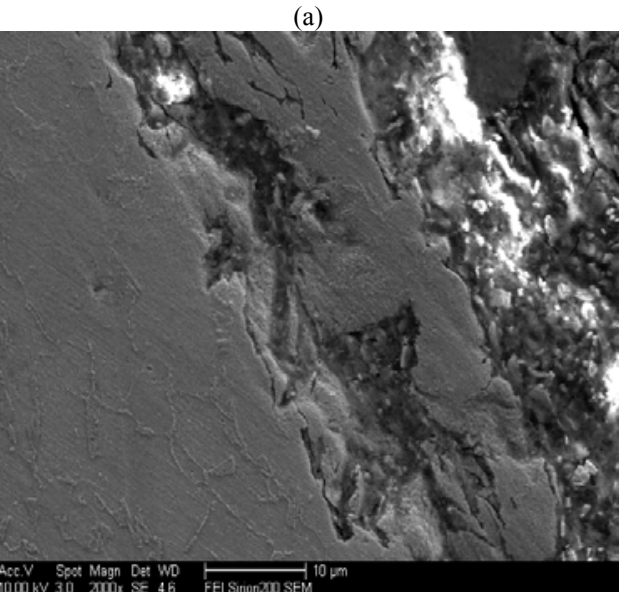

(b)

FIGURE IV. THE SEM PHOTOGRAPH OF THE ZINC COATING SURFACE AND CROSS SECTION

\section{CONCLUSIONS}

A novel wet ultrasonic mechanical plating method was presented. The theory was studied and the ultrasonic mechanical plating equipment was designed. Ultrasonic transducer and amplitude transformer horn were designed on the basis of calculation. Galvanized tests were carried out using the equipment. The zinc coating layer was tested after plating. The results show that the coating layer is smooth, even and compact. Its maximum thickness can reach $30 \mu \mathrm{m}$. The bonding strength between the coating and substrate is satisfactory.

\section{ACKNOWLEDGMENTS}

The work described in this paper is supported by Natural Science Foundation of Shandong Province. (Subject number: ZR2015EM053). 


\section{REFERENCES}

[1] Wang Shengmin, Zhao Xiaojun, He Mingyi, Research status and development of mechanical plating, Materials Review, 31(2017)117-122.

[2] Wang Shengmin, Liu li, Zhao Xiaojun, He mingyi, Cleaner production of mechanical zinc-plating, Techniques and equipment for environment pollution control, v(2003)83-85

[3] Sergey V. Komarov, Sang H. son, Naohito Hayashi, Sergey D. Kaloshkin, Oleg V. Abramov, Eiki Kasai, Development of a novel method for mechanical plating using ultrasonic vibrations, Surface \& Coatings Technology, 201(2007)6999-7006.

[4] Komarov Sergey V., Son Sang Han, Kaloshkin Sergey D., Kasai Eiki, A dry ultrasonic-based method for mechanical coating, Reviews on Advanced Materials Science, v(2008)691-696.

[5] Li Guihua, Zhang Xianghui, Fu Shuigen, Zuo Jing, Gao Ju, Design of ultrasonic transducer with a quarter wavelength composite exponential horn, Machinery Design \& Manufacture, 5(2009)1-3. 